\title{
Finite speed of propagation in porous media by mass transportation methods ${ }^{\star}$
}

\author{
José Antonio Carrillo ${ }^{\mathrm{a}}$, Maria Pia Gualdani ${ }^{\mathrm{b}}$, Giuseppe Toscani ${ }^{\mathrm{c}}$ \\ ${ }^{a}$ Departament de Matemàtiques - ICREA, Universitat Autònoma de Barcelona, E-08193 Bellaterra, Spain \\ ${ }^{\mathrm{b}}$ Fachbereich Mathematik, Universität Mainz, Staudingerweg 9, D-55099 Mainz, Germany \\ ${ }^{\mathrm{c}}$ Dipartimento di Matematica, Universitá di Pavia, via Ferrata 1, I-27100 Pavia, Italy
}

\begin{abstract}
In this note we make use of mass transportation techniques to give a simple proof of the finite speed of propagation of the solution to the one-dimensional porous medium equation. The result follows by showing that the difference of support of any two solutions corresponding to different compactly supported initial data is a bounded in time function of a suitable Monge-Kantorovich related metric. To cite this article: J. A. Carrillo, M. P. Gualdani, G. Toscani, C. R. Acad. Sci. Paris, Ser. I 336 (2003).

\section{Résumé}

Dans cette note nous utilisons des techniques de transport de masse pour donner une preuve élémentaire de la finitude de la vitesse de propagation des solutions de l'équation mono-dimensionnelle des milieux poreux. Le résultat repose sur la preuve de la propriété suivante : la différence du support entre deux solutions quelconques correspondant à des données initiales à support compact différentes est une fonction, bornée en temps, d'une métrique de Monge-Kantorovitch appropriée. Pour citer cet article : J. A. Carrillo, M. P Gualdani, G. Toscani, C. R. Acad. Sci. Paris, Ser. I 336 (2003).
\end{abstract}

\section{Introduction}

We consider the problem

$$
\begin{aligned}
& u_{t}=\left(u^{m}\right)_{x x}, \quad x \in \mathbb{R}, \quad t>0, \quad m>1, \\
& u(x, 0)=u_{0}(x), \quad x \in \mathbb{R},
\end{aligned}
$$

\footnotetext{
* Work partially supported by EEC network \#HPRN-CT-2002-00282, by the bilateral project Azioni integrate ItaliaSpagna, by the Vigoni Project CRUI-DAAD and by the Spanish DGI-MCYT/FEDER project BFM2002-01710.

Email addresses: carrillo@mat.uab.es (José Antonio Carrillo), gualdani@mathematik.uni-mainz.de (Maria Pia Gualdani), toscani@dimat.unipv.it (Giuseppe Toscani).
} 
where $u_{0} \in L^{1}(\mathbb{R}) \cap L^{\infty}(\mathbb{R}), u_{0} \geq 0$ and $u_{0}$ is compactly supported.

Much is already known for problem (1)-(2): see $[1,2,3,4,5]$ and the references therein for existence, uniqueness and asymptotic behaviour results of the porous media equation. It also known that the degeneracy at level $u=0$ of the diffusivity $D(u)=m u^{m-1}$ causes the phenomenon called finite speed of propagation. This means that the support of the solution $u(\cdot, t)$ to $(1)-(2)$ is a bounded set for all $t \geq 0$. In fact it can be proved that the solution $u(x, t)$ as $t \rightarrow+\infty$ converges to the Barenblatt source-type solution $U(x, t, C)$ with the same mass as the initial data.

In this paper we want to give a simple proof of the finite propagation property using mass transportation techniques. Precisely, we prove that the difference of support of two solutions of (1)-(2) with different compactly supported initial conditions is a bounded in time function of a suitable Monge-Kantorovich related metric.

Theorem 1.1 Let $u_{1}(x, t)$ and $u_{2}(x, t)$ be strong solutions of (1)-(2) with initial conditions $u_{01}(x)$ and $u_{02}(x)$ respectively, where $u_{0 i} \in L^{1}(\mathbb{R}) \cap L^{\infty}(\mathbb{R}), u_{0 i} \geq 0$ and $u_{0 i}$ is compactly supported, $i=1$, 2 , and let $\Omega_{i}=\left\{(x, t) \in \mathbb{R} \times[0,+\infty) / u_{i}(x, t)>0\right\}, \quad i=1,2$.

Let $\xi_{i}(t)=\inf _{x \in \mathbb{R}} \Omega_{i}, \Xi_{i}(t)=\sup _{x \in \mathbb{R}} \Omega_{i}$, for $t \geq 0, i=1,2$. Then

$$
\max \left\{\left|\xi_{1}(t)-\xi_{2}(t)\right|,\left|\Xi_{1}(t)-\Xi_{2}(t)\right|\right\} \leq W_{\infty}\left(u_{01}, u_{02}\right), \quad \forall t \in[0,+\infty),
$$

where $W_{\infty}\left(u_{01}, u_{02}\right)$ is a constant, which depends only on the initial data $u_{01}, u_{02}$ and is defined in (16). The finite speed of propagation property follows by just taking as one of the solutions a time translation of the explicit Barenblatt solution which is known to have compact support expanding at the rate $t^{1 /(m+1)}$.

\section{Proof}

Consider a sequence of functions $u_{n} \in C^{\infty}([0,+\infty) \times \mathbb{R})$, which are strong solutions (see [3]) of the problems $P_{n}$

$$
\begin{aligned}
& u_{t}=\left(u^{m}\right)_{x x}, \quad x \in \mathbb{R}, \quad t>0, \quad m>1, \\
& u(x, 0)=u_{0 n}(x), \quad x \in \mathbb{R},
\end{aligned}
$$

where $u_{0 n}(x), n \in \mathbb{N}$, is a sequence of bounded, integrable and strictly positive $C^{\infty}$-smooth functions such that all their derivatives are bounded in $\mathbb{R}$, the condition $(m-1)\left(u_{0}^{m}\right)_{x x} \geq-a u_{0 n}$ holds for some constant $a>0$, and $u_{0 n} \rightarrow u_{0}$ in $L^{1}(\mathbb{R})$ if $n \rightarrow+\infty$. We may always do it in such a way that $\left\|u_{0 n}\right\|_{L^{1}(\mathbb{R})}=\left\|u_{0}\right\|_{L^{1}(\mathbb{R})}$ and $\left\|u_{0 n}\right\|_{L^{\infty}(\mathbb{R})} \leq\left\|u_{0}\right\|_{L^{\infty}(\mathbb{R})}$. From the $L^{1}$-contraction property it follows that $u_{n} \rightarrow u$ in $C\left([0,+\infty): L^{1}(\mathbb{R})\right)$ if $\quad n \rightarrow+\infty$, where $u$ is a strong solution of (1)-(2) (see [3], chapt. III).

This sequence of regularized solutions can be further approximated by a sequence of initial boundary value problems. We introduce a cutoff sequence $\theta_{k} \in C^{\infty}(\mathbb{R}), 1<k \in \mathbb{N}$, with the following properties:

$$
\theta_{k}(x)=1 \quad \text { for } \quad|x|<k-1, \quad \theta_{k}(x)=0 \quad \text { for } \quad|x| \geq k, \quad 0<\theta_{k}<1 \quad \text { for } \quad k-1<|x|<k .
$$

The initial boundary value problem $P_{n k}$

$$
\begin{aligned}
& u_{t}=\left(u^{m}\right)_{x x}, \quad x \in(-k, k), \quad t>0, \\
& u(x, 0)=u_{0 n k}(x):=\frac{u_{0 n}(x) \theta_{k}(x)}{\left\|u_{0 n}(x) \theta_{k}(x)\right\|_{L^{1}}}, \\
& u(x, t)=0 \quad \text { for } \quad|x|=k, \quad t \geq 0,
\end{aligned}
$$


is mass preserving and has a unique solution $u_{n k}(x, t) \in C^{\infty}((0,+\infty) \times[-k, k]) \cap C([0,+\infty) \times[-k, k])$, strictly positive for $x \in(-k, k)$ and zero at the boundary (see [3], prop.6, chapt.II). Because $u_{0 n k} \longrightarrow u_{0 n}$ as $k \longrightarrow+\infty$, for all $n \in \mathbb{N}, u_{n k} \rightarrow u_{n} \quad$ in $\quad C\left([0,+\infty): L^{1}(\mathbb{R})\right)$ if $k \rightarrow+\infty$, where $u_{n}$ is solution of the problem $P_{n}$.

Thanks to estimates independent of $k$ for the moments of the solutions of the $P_{n k}$ problems and passing to the limit in the corresponding inequalities, it can be easily shown that the solution $u_{n}(x, t)$ of $(4)-(5)$ enjoys an important property. It holds

$$
\int_{\mathbb{R}}|x|^{p} u_{n}(x, t) d x<+\infty, \quad \forall t \geq 0, \quad \forall p \in[1,+\infty) .
$$

We shall denote by $\mathbb{P}_{p}(\mathbb{R})$, with $p \in[1,+\infty)$, the set of all probability measures on $\mathbb{R}$ with finite moments of order $p$. Let $\Pi(\mu, \nu)$ be the set of all probability measures on $\mathbb{R}^{2}$ having $\mu, \nu \in \mathbb{P}_{p}(\mathbb{R})$ as marginal distributions (see [6]). The Wasserstein $p$-distance between two probability measures $\mu, \nu \in$ $\mathbb{P}_{p}(\mathbb{R})$ is defined as

$$
W_{p}(\mu, \nu)^{p}:=\inf _{\pi \in \Pi(\nu, \mu)} \int_{\mathbb{R}^{2}}|x-y|^{p} d \pi(x, y), \quad \forall p \in[1,+\infty) .
$$

$W_{p}$ defines a metric on $\mathbb{P}_{p}(\mathbb{R})$ (see $[6]$ ). Bound (10) then shows that the Wasserstein $p$-distance between any two solutions which is initially finite, remains finite at any subsequent time.

Any probability measure $\mu$ on the real line can be described in terms of its cumulative distribution function $F(x)=\mu((-\infty, x])$ which is a right-continuous and non-decreasing function with $F(-\infty)=0$ and $F(+\infty)=1$. Then, the generalized inverse of $F$ defined by $F^{-1}(\eta)=\inf \{x \in \mathbb{R} / F(x)>\eta\}$ is also a right-continuous and non-decreasing function on $[0,1]$.

Let $\mu, \nu \in \mathbb{P}_{p}(\mathbb{R})$ be probability measures and let $F(x), G(x)$ be the respective distribution functions. On the real line (see [6]), the value of the Wasserstein $p$-distance $W_{p}(\mu, \nu)$ can be explicitly written in terms of the generalized inverse of the distribution functions,

$$
W_{p}(\mu, \nu)^{p}=\int_{0}^{1}\left|F^{-1}(\eta)-G^{-1}(\eta)\right|^{p} d \eta, \quad \forall p \in[1,+\infty) .
$$

Let $u_{1}(x, t), u_{2}(x, t)$ be strong solutions of (1)-(2) corresponding to initial conditions $u_{01}(x)$ and $u_{02}(x)$ respectively. We denote by $u_{1_{n}}(x, t)$ and $u_{2 n}(x, t)$ the strong solutions of (4)-(5) with initial conditions $u_{01 n}(x)$ and $u_{02 n}(x)$ respectively, where $u_{0 i n} \longrightarrow u_{0 i}$ in $L^{1}(\mathbb{R})$ for $i=1,2$. Analogously, we consider the solutions $u_{1_{n k}}(x, t)$ and $u_{2 n k}(x, t)$ of the problems $P_{n k}$ converging towards $u_{i n}(x, t)$ for $i=1,2$ in $C\left([0,+\infty): L^{1}(\mathbb{R})\right)$ as $k \rightarrow \infty$.

Let $F_{i_{n k}}(x, t)$ be the distribution functions of $u_{i_{n k}}$ for $i=1,2$. A direct computation shows that $F_{i_{n k}}^{-1}(\eta, t)$ solves the following equation

$$
\frac{\partial F_{i n k}^{-1}}{\partial t}=-\frac{\partial}{\partial \eta}\left(\left(\frac{\partial F_{i n k}^{-1}}{\partial \eta}\right)^{-m}\right), \quad i=1,2
$$

for $t>0$ and $\eta \in[0,1]$. Making use of equation (13), it is easy to prove that the Wasserstein $p$-distance

$$
W_{p}\left(u_{1 n k}, u_{2 n k}\right)(t)=\left\{\int_{0}^{1}\left|F_{1 n k}^{-1}(\eta, t)-F_{2 n k}^{-1}(\eta, t)\right|^{p} d \eta\right\}^{\frac{1}{p}}, \quad \forall p \in[1,+\infty),
$$


is a non-increasing in time function. In fact, for any given $p \geq 1$, integrating by parts one obtains

$$
\begin{aligned}
& \frac{d}{d t} \int_{0}^{1}\left|F_{1}^{-1}(\eta, t)-F_{2 k}^{-1}(\eta, t)\right|^{p} d \eta=p(p-1) \int_{0}^{1}\left|F_{1_{n k}}^{-1}(\eta, t)-F_{2 n k}^{-1}(\eta, t)\right|^{p-2} \\
& \times\left(F_{1_{n k}}^{-1}(\eta, t)_{\eta}-F_{2 n k}^{-1}(\eta, t)_{\eta}\right)\left[\left(F_{1_{n k}}^{-1}(\eta, t)_{\eta}\right)^{-m}-\left(F_{2 n k}^{-1}(\eta, t)_{\eta}\right)^{-m}\right] d \eta \leq 0
\end{aligned}
$$

since the function $x^{-m}, m \geq 1$, is decreasing. Note that the boundary terms vanish due to the compact support of the solutions, which implies

$$
\lim _{\eta \rightarrow 0^{+}}\left(F_{i_{n k}}^{-1}(\eta, t)_{\eta}\right)^{-1}=\lim _{\eta \rightarrow 1^{-}}\left(F_{i_{n k}}^{-1}(\eta, t)_{\eta}\right)^{-1}=0 \quad i=1,2 .
$$

On the other hand, for all $p \in[1,+\infty)$,

$$
W_{p}\left(u_{1 n k}, u_{2 n k}\right) \rightarrow W_{p}\left(u_{1 n}, u_{2 n}\right), \quad k \rightarrow+\infty, \quad W_{p}\left(u_{1 n}, u_{2 n}\right) \rightarrow W_{p}\left(u_{1}, u_{2}\right), \quad n \rightarrow+\infty .
$$

This implies that $W_{p}\left(u_{1}, u_{2}\right) \leq W_{p}\left(u_{01}, u_{02}\right), \forall p \in[1,+\infty)$. Since the function $W_{p}\left(u_{1}, u_{2}\right)$ is increasing with respect to $p$, we can define the quantity

$$
W_{\infty}\left(u_{1}, u_{2}\right):=\lim _{p \uparrow+\infty} W_{p}\left(u_{1}, u_{2}\right)=\sup _{\eta \in(0,1)} \operatorname{ess}\left|F_{1}^{-1}(\eta, t)-F_{2}^{-1}(\eta, t)\right| .
$$

Since $W_{\infty}\left(u_{01}, u_{02}\right)$ is finite, we deduce easily that $W_{\infty}\left(u_{1}, u_{2}\right)$ is also a non-increasing in time function. Note that the inverse function $F^{-1}(\eta)$ of a distribution $F(x)=\int_{-\infty}^{x} u(s) d s$, where $u(s)$ is a integrable compactly supported function, is continuous at the point $\eta=0$ and $\eta=1$. Thus we can justify the inequality

$$
\begin{gathered}
W_{\infty}\left(u_{1}, u_{2}\right)=\sup _{\eta \in(0,1)} \operatorname{ess}\left|F_{1}^{-1}(\eta, t)-F_{2}^{-1}(\eta, t)\right| \geq \\
\max \left\{\left|F_{1}^{-1}(0, t)-F_{2}^{-1}(0, t)\right|,\left|F_{1}^{-1}(1, t)-F_{2}^{-1}(1, t)\right|\right\} \geq \max \left\{\left|\xi_{1}(t)-\xi_{2}(t)\right|,\left|\Xi_{1}(t)-\Xi_{2}(t)\right|\right\} .
\end{gathered}
$$

We remark that the above arguments only hold in one space dimension due to the fact that only in this case one can express the $p$-Wasserstein distance in terms of pseudo-inverse distribution functions, as given in (12).

\section{References}

[1] J.A. Carrillo, G. Toscani. Asymptotic $L^{1}$-decay of solutions of the Porous Medium equation to self-similarity. Indiana University Mathematics Journal, 49, 1 (2000), 113-142

[2] A. S. Kalashnikov. Some problems of the qualitative theory of non-linear degenerate second-order parabolic equations. Russian Math. Surveys 42, 2 (1987), 169-222.

[3] J. L. Vázquez. An Introduction to the mathematical theory of the porous medium equation. Shape Optimization and Free Boundaries (Montreal, PQ, 1990), 347-389, NATO Adv. Sci. Inst. Ser. C Math. Phys. Sci., 380, Kluwer Acad. Publ., Dordrecht, 1992

[4] J. L. Vázquez. Asymptotic behaviour for the porous medium equation posed in the whole space. J. Evol. Equ. 3 (2003), $67-118$

[5] J. L. Vázquez. Asymptotic behaviour and propagation properties of the one-dimensional flow of gas in a porous medium. Transactions of the american mathematical society, 277, 2, June 1983

[6] C. Villani. Topics in mass transportation. Graduate Studies in Mathematics 58, AMS ISSN: 1065-7339, 2003. 